\title{
Forced Oscillation of Solutions of a Fractional Neutral Partial Functional Differential Equation
}

\section{Sadhasivam, J. Kavitha*}

Post Graduate and Research Department of Mathematics, Thiruvalluvar Government Arts College, Rasipuram, Namakkal Dt. Tamil Nadu, India

Email: ovsadha@gmail.com, ${ }^{*}$ kaviakshita@gmail.com

Received 16 June 2015; accepted 20 July 2015; published 24 July 2015

Copyright (C) 2015 by authors and Scientific Research Publishing Inc.

This work is licensed under the Creative Commons Attribution International License (CC BY).

http://creativecommons.org/licenses/by/4.0/

(c) () Open Access

\section{Abstract}

In this paper, we will establish the sufficient conditions for the oscillation of solutions of neutral time fractional partial differential equation of the form

$$
\begin{aligned}
& D_{+, t}^{\alpha}\left[p(t) D_{+, t}^{\alpha}\left(u(x, t)+\sum_{i=1}^{n} c_{i}(t) u\left(x, t-\tau_{i}\right)\right)\right]+q(x, t) u(x, t)+\sum_{j=1}^{m} q_{j}(x, t) f_{j}\left(u\left(x, t-\sigma_{j}\right)\right) \\
& =a(t) \Delta u(x, t)+\sum_{k=1}^{s} a_{k}(t) \Delta u\left(x, t-\rho_{k}\right)+F(x, t)
\end{aligned}
$$

for $(x, t) \in \Omega \times R_{+}=G, R_{+}=[0, \infty)$, where $\Omega$ is a bounded domain in $R^{N}$ with a piecewise smooth boundary $\partial \Omega, \alpha \in(0,1)$ is a constant, $D_{+, t}^{\alpha}$ is the Riemann-Liouville fractional derivative of order $\alpha$ of $u$ with respect to $t$ and $\Delta$ is the Laplacian operator in the Euclidean $N$-space $R^{N}$ subject to the condition $\int_{t_{0}}^{\infty} \frac{\mathrm{d} t}{p(t)}<\infty, t_{0} \geq 0 . \frac{\partial u(x, t)}{\partial \gamma}+g(x, t) u(x, t)=0, \quad(x, t) \in \partial \Omega \times R_{+}$.

\section{Keywords}

Fractional, Neutral, Oscillation, Partial, Functional

\section{Introduction}

Fractional differential equations are generalizations of classical differential equations to an arbitrary non integer *Corresponding author. 
order and have gained considerable importance due to the fact that these equations are applied in real world problems arising in various branches of science and technology [1]-[5]. Neutral delay differential equations have applications in electric networks containing Lossless transmission lines and population dynamics [6]. Several papers concerning neutral parabolic differential equations have appeared recently (for example see [7] [8]). The oscillatory theory of solutions of fractional differential equations has received a great deal of attention [9]-[15]. In the last few years, many authors studied the oscillation of a time-fractional partial differential equations [16] [17]. There are only few works has been done on oscillation of forced neutral fractional partial differential equations.

In this paper, we study the oscillatory behavior of solutions of nonlinear neutral fractional differential equations with forced term of the form

$$
\begin{aligned}
& D_{+, t}^{\alpha}\left[p(t) D_{+, t}^{\alpha}\left(u(x, t)+\sum_{i=1}^{n} c_{i}(t) u\left(x, t-\tau_{i}\right)\right)\right]+q(x, t) u(x, t)+\sum_{j=1}^{m} q_{j}(x, t) f_{j}\left(u\left(x, t-\sigma_{j}\right)\right) \\
& =a(t) \Delta u(x, t)+\sum_{k=1}^{s} a_{k}(t) \Delta u\left(x, t-\rho_{k}\right)+F(x, t), \quad(x, t) \in \Omega \times R_{+}=G,
\end{aligned}
$$

where $\Omega$ is a bounded domain in $R^{N}$ with a piecewise smooth boundary $\partial \Omega, \alpha \in(0,1)$ is a constant, $D_{+, t}^{\alpha}$ is the Riemann-Liouville fractional derivative of order $\alpha$ of $u$ with respect to $t$ and $\Delta$ is the Laplacian operator in the Euclidean N-space $R^{N}$ (ie) $\Delta u(x, t)=\sum_{r=1}^{N}\left(\partial^{2} u(x, t) / \partial x_{r}^{2}\right)$. Equation (E) is supplemented with the boundary condition

$$
\left(\mathrm{B}_{1}\right) \frac{\partial u(x, t)}{\partial \gamma}+g(x, t) u(x, t)=0,(x, t) \in \partial \Omega \times R_{+},
$$

where $\gamma$ is the unit exterior normal vector to $\partial \Omega$ and $g(x, t)$ is non negative continuous function on $\partial \Omega \times R_{+}$, and

(B) $u(x, t)=0,(x, t) \in \partial \Omega \times R_{+}$.

In what follows, we always assume without mentioning that

$\left(\mathrm{A}_{1}\right) \quad p \in C^{\alpha}\left((0, \infty) ; R_{+}\right)$such that $\int_{t_{0}}^{\infty} \frac{\mathrm{d} t}{p(t)}<\infty$;

$\left(\mathrm{A}_{2}\right) \quad c_{i} \in C^{2 \alpha}\left((0, \infty) ; R_{+}\right), 0 \leq \sum_{i=1}^{n} c_{i}<1$, and $\tau=\max \left\{\tau_{1}, \tau_{2}, \cdots, \tau_{n}\right\}, \quad \tau_{i}$ are non negative constants, $i \in I_{n}=\{1,2, \cdots, n\}$;

$\left(\mathrm{A}_{3}\right) \quad q, q_{j} \in C\left(\bar{G} ; R_{+}\right)$and $q(t)=\min _{x \in \bar{\Omega}} q(x, t), q_{j}(t)=\min _{x \in \bar{\Omega}} q_{j}(x, t), j \in I_{m}=\{1,2, \cdots, m\}$;

$\left(\mathrm{A}_{4}\right) a, a_{k} \in C\left((0, \infty) ; R_{+}\right), \rho_{k}$ and $\sigma_{j}$ are nonnegative constants, $j \in I_{m}=\{1,2, \cdots, m\} ; k \in I_{s}=\{1,2, \cdots, s\}$;

$\left(\mathrm{A}_{5}\right) f_{j} \in C(R ; R)$ are convex in $(0, \infty)$, and $u f_{j}(u)>0$ for $u \neq 0, j \in I_{m}$;

( $\left.\mathrm{A}_{6}\right) \quad F \in C\left(\bar{G} ; R_{+}\right)$such that $\int_{\Omega} F(x, t) \mathrm{d} x \leq 0$.

A function $u \in C^{2 \alpha}(G) \cap C^{\alpha}(\bar{G})$ is called a solution of $(\mathrm{E}),\left(\mathrm{B}_{1}\right)\left((\mathrm{E}),\left(\mathrm{B}_{2}\right)\right)$ if it satisfies in the domain $G$ and the boundary condition $\left(\mathrm{B}_{1}\right),\left(\mathrm{B}_{2}\right)$. The solution of $u(x, t)$ of equations $(\mathrm{E}),\left(\mathrm{B}_{1}\right)$ or $(\mathrm{E}),\left(\mathrm{B}_{2}\right)$ is said to be oscillatory in the domain $G$ if for any positive number $\mu$ there exists a point $\left(x_{0}, t_{0}\right) \in \Omega \times[\mu, \infty)$ such that $u\left(x_{0}, t_{0}\right)=0$ holds. Particularly no work has been known with $(\mathrm{E})$ and $\left(\mathrm{B}_{1}\right)$ up to now. To develop the qualitative properties of fractional partial differential equations, it is very interesting to study the oscillatory behavior of (E) and $\left(\mathrm{B}_{1}\right)$. The purpose of this paper is to establish some new oscillation criteria for (E) by using a generalized Riccati technique and integral averaging technique. Our results are essentially new.

\section{Preliminaries}

In this section, we give the definitions of fractional derivatives and integrals and some notations which are useful throughout this paper. There are several kinds of definitions of fractional derivatives and integrals. In this paper, we use the Riemann-Liouville left sided definition on the half-axis $R_{+}$. The following notations will be used for the convenience.

$$
v(t)=\frac{1}{|\Omega|} \int_{\Omega} u(x, t) \mathrm{d} x \text {, where }|\Omega|=\int_{\Omega} \mathrm{d} x,
$$




$$
\begin{aligned}
& \xi=\frac{t^{\alpha}}{\Gamma(1+\alpha)}, \quad \tilde{p}(\xi)=p(t), \tilde{W}(\xi)=W(t) \\
& \tilde{A}(\xi)=A(t), \tilde{Q}(\xi)=Q(t), \xi_{0}=\frac{t_{0}^{\alpha}}{\Gamma(1+\alpha)}, \quad \xi_{1}=\frac{t_{1}^{\alpha}}{\Gamma(1+\alpha)} .
\end{aligned}
$$

Definition 2.1. The Riemann-Liouville fractional partial derivative of order $0<\alpha<1$ with respect to $t$ of a function $u(x, t)$ is given by

$$
\left(D_{+, t}^{\alpha} u\right)(x, t):=\frac{\partial}{\partial t} \frac{1}{\Gamma(1-\alpha)} \int_{0}^{t}(t-v)^{-\alpha} u(x, v) \mathrm{d} v
$$

provided the right hand side is point wise defined on $R_{+}$where $\Gamma$ is the gamma function.

Definition 2.2. The Riemann-Liouville fractional integral of order $\alpha>0$ of a function $y: R_{+} \rightarrow R$ on the half-axis $R_{+}$is given by

$$
\left(I_{+}^{\alpha} y\right)(t):=\frac{1}{\Gamma(\alpha)} \int_{0}^{t}(t-v)^{\alpha-1} y(v) \mathrm{d} v \text { for } t>0
$$

provided the right hand side is pointwise defined on $R_{+}$.

Definition 2.3. The Riemann-Liouville fractional derivative of order $\alpha>0$ of a function $y: R_{+} \rightarrow R$ on the half-axis $R_{+}$is given by

$$
\left(D_{+}^{\alpha} y\right)(t):=\frac{\mathrm{d}^{\lceil\alpha\rceil}}{\mathrm{d} t^{\lceil\alpha\rceil}}\left(I_{+}^{\lceil\alpha\rceil-\alpha} y\right)(t) \quad \text { for } t>0
$$

provided the right hand side is pointwise defined on $R_{+}$where $\lceil\alpha\rceil$ is the ceiling function of $\alpha$.

Lemma 2.1. Let $y$ be the solution of (E) and

$$
K(t):=\int_{0}^{t}(t-v)^{-\alpha} y(v) \mathrm{d} v \quad \text { for } \alpha \in(0,1) \text { and } t>0 .
$$

Then $K^{\prime}(t)=\Gamma(1-\alpha)\left(D_{+}^{\alpha} y\right)(t)$.

\section{Oscillation of $(\mathrm{E}),\left(\mathrm{B}_{1}\right)$}

We introduce a class of function P. Let

$$
D_{0}=\left\{(t, s): t>s \geq t_{0}\right\} \text { and } D=\left\{(t, s): t \geq s \geq t_{0}\right\} .
$$

The function $H \in C(D, R)$ is said to belong to the class $P$, if

$\left.\mathrm{C}_{1}\right) H(t, t)=0$ for $t \geq t_{0}, H(t, s)>0$ for $(t, s) \in D_{0}$;

$\left.\mathrm{C}_{2}\right) \quad H$ has a continuous and non-positive partial derivative $\frac{\partial H(t, s)}{\partial s}$ on $D_{0}$ with respect to $s$.

Lemma 3.1. If $u(x, t)$ is a solution of $(\mathrm{E})$, $\left(\mathrm{B}_{1}\right)$ for which $u(x, t)>0$ in $G_{T}=\Omega \times[T, \infty), T \geq 0$, then the function $v(t)$ is defined by (1) satisfy the fractional differential inequality

$$
D_{+}^{\alpha}\left[p(t) D_{+}^{\alpha}\left(v(t)+\sum_{i=1}^{n} c_{i}(t) v\left(t-\tau_{i}\right)\right)\right]+q(t) v(t)+\sum_{j=1}^{m} q_{j}(t) f_{j}\left(v\left(t-\sigma_{j}\right)\right) \leq 0
$$

with $v(t)>0, v\left(t-\tau_{i}\right)>0, i \in I_{n}$ and $v\left(t-\sigma_{j}\right)>0, j \in I_{m}$ for $t \geq T$.

Proof. Let $t \geq T$. Integrating (E) with respect to $x$ over $\Omega$, we have

$$
\begin{aligned}
& \int_{\Omega} D_{+, t}^{\alpha}\left[p(t) D_{+, t}^{\alpha}\left(u(x, t)+\sum_{i=1}^{n} c_{i}(t) u\left(x, t-\tau_{i}\right)\right)\right] \mathrm{d} x+\int_{\Omega} q(x, t) u(x, t) \mathrm{d} x+\sum_{j=1}^{m} \int_{\Omega} q_{j}(x, t) f_{j}\left(u\left(x, t-\sigma_{j}\right)\right) \mathrm{d} x \\
& =a(t) \int_{\Omega} \Delta u(x, t) \mathrm{d} x+\sum_{k=1}^{s} a_{k}(t) \int_{\Omega} \Delta u\left(x, t-\rho_{k}\right) \mathrm{d} x+\int_{\Omega} F(x, t) \mathrm{d} x .
\end{aligned}
$$


Using Green's formula and boundary condition $\left(\mathrm{B}_{1}\right)$ it follows that

$$
\int_{\Omega} \Delta u(x, t) \mathrm{d} x=\int_{\partial \Omega} \frac{\partial u(x, t)}{\partial \gamma} \mathrm{d} S=-\int_{\partial \Omega} g(x, t) u(x, t) \mathrm{d} S \leq 0, \quad t \geq T
$$

and

$$
\int_{\Omega} \Delta u\left(x, t-\rho_{k}\right) \mathrm{d} x=\int_{\partial \Omega} \frac{\partial u\left(x, t-\rho_{k}\right)}{\partial \gamma} \mathrm{d} S=-\int_{\partial \Omega} g\left(x, t-\rho_{k}\right) u\left(x, t-\rho_{k}\right) \mathrm{d} S \leq 0, \quad t \geq T, \quad k \in I_{s} .
$$

Also from $\left(A_{3}\right),\left(A_{5}\right)$, we obtain

$$
\int_{\Omega} q(x, t) u(x, t) \mathrm{d} x \geq q(t) \int_{\Omega} u(x, t) \mathrm{d} x, t \geq T
$$

and using and Jensen's inequality we get

$$
\begin{aligned}
& \int_{\Omega} q_{j}(x, t) f_{j} u\left(x, t-\sigma_{j}\right) \mathrm{d} x \\
& \geq q_{j}(t) \int_{\Omega} f_{j}\left(u\left(x, t-\sigma_{j}\right)\right) \mathrm{d} x \geq q_{j}(t) \int_{\Omega} \mathrm{d} x f_{j}\left(\int_{\Omega} u\left(x, t-\sigma_{j}\right) \mathrm{d} x\right)\left(\int_{\Omega} \mathrm{d} x\right)^{-1}, t \geq T .
\end{aligned}
$$

In view of (1), (7)-(10) and $\mathrm{A}_{6}$, (6) yield

$$
D_{+}^{\alpha}\left[p(t) D_{+}^{\alpha}\left[v(t)+\sum_{i=1}^{n} c_{i}(t) v\left(t-\tau_{i}\right)\right]\right]+q(t) v(t)+\sum_{j=1}^{m} q_{j}(t) f_{j}\left(v\left(t-\sigma_{j}\right)\right) \leq 0, \quad t \geq T .
$$

This completes the proof.

Lemma 3.2. Let $u(x, t)$ be a positive solution of the (E), ( $\left.\mathrm{B}_{1}\right)$ defined on $G_{T}=\Omega \times[T, \infty), T \geq 0$ then the function $z(t)=v(t)+\sum_{i=1}^{n} c_{i}(t) v\left(t-\tau_{i}\right)$ where $v(t)$ is defined by (1) satisfies one of the following conditions:

1) $z(t)>0, D_{+}^{\alpha} z(t)>0, D_{+}^{\alpha}\left(p(t) D_{+}^{\alpha} z(t)\right) \leq 0$,

2) $z(t)>0, D_{+}^{\alpha} z(t)<0, D_{+}^{\alpha}\left(p(t) D_{+}^{\alpha} z(t)\right) \leq 0$, for all $t \geq T$.

Proof. From Lemma 3.1, the function $v(t)$ satisfies the inequality (5) and $v(t)>0, v\left(t-\tau_{i}\right)>0, i \in I_{n}$ and $v\left(t-\sigma_{j}\right)>0, j \in I_{m}$ for $t \geq T$. From (5) and the hypothesis we have $z(t)>0$ and $D_{+}^{\alpha}\left(p(t) D_{+}^{\alpha} z(t)\right) \leq 0$ for $t \geq T$. Hence $\left(p(t) D_{+}^{\alpha} z(t)\right)$ is monotonic and eventually of one sign. This completes the proof.

Lemma 3.3. Let $u(x, t)$ be a positive solution of (E), $\left(\mathrm{B}_{1}\right)$ defined on $G_{T}$ and suppose Case (1) of Lemma 3.2 holds, then

$$
v(t) \geq\left(1-\sum_{i=1}^{n} c_{i}(t)\right) z(t) \text {, for } t \geq T \geq t_{0} .
$$

Proof. From Case (I), $z(t)$ is positive and increasing for $t \geq T$, and by the definition of $z(t)$, we obtain $z(t) \geq v(t)$ and

$$
v(t)=z(t)-\sum_{i=1}^{n} c_{i}(t) v\left(t-\tau_{i}\right) \geq z(t)-\sum_{i=1}^{n} c_{i}(t) z\left(t-\tau_{i}\right) \geq\left(1-\sum_{i=1}^{n} c_{i}(t)\right) z(t) \text { for } t \geq T .
$$

This completes the proof.

Lemma 3.4. Let $u(x, t)$ be a positive solution of $(E),\left(B_{1}\right)$ defined on $G_{T}$ and suppose Case (2) of Lemma 3.2 holds, then

$$
v(t-\tau) \geq \frac{z(t)}{\left(1+\sum_{i=1}^{n} c_{i}(t)\right)} \geq\left(1-\sum_{i=1}^{n} c_{i}(t)\right) z(t), \text { for } t \geq T .
$$

Proof. In this case the function $z(t)$ is positive and nonincreasing for $t \geq T$ and therefore without loss of 
generality we may assume from the definition of $z(t)$ and $v(t)$ is also nonincreasing for $t \geq T$. Hence $z(t)=v(t)+\sum_{i=1}^{n} c_{i}(t) v\left(t-\tau_{i}\right) \leq v(t-\tau)+\sum_{i=1}^{n} c_{i}(t) v(t-\tau)$ which implies (12).

This completes the proof.

Theorem 3.1. Assume that $\frac{f_{j}(u)}{u} \geq \alpha_{j}$ for $u \neq 0$, where $\alpha_{j}$ are positive constants $j \in I_{m}$. Let $h, H: D \rightarrow R$ be continuous functions such that $H \in P$ and

$$
-\frac{\partial H}{\partial s}(\xi, s)=h(\xi, s) \sqrt{H(\xi, s)} \text { for all }(\xi, s) \in D_{0} .
$$

Assume also that there exists a positive nondecreasing function $\rho \in C^{\alpha}\left(\left[t_{0}, \infty\right) ; R_{+}\right)$such that

$$
\limsup _{\xi \rightarrow \infty} \frac{1}{H\left(\xi, \xi_{0}\right)} \int_{\xi_{0}}^{\xi}\left[H(\xi, s) \tilde{A}(s) \tilde{\rho}(s)-\frac{\tilde{\rho}(s) \tilde{p}(s-\sigma) \tilde{Q}^{2}(\xi, s)}{4}\right] \mathrm{d} s=\infty,
$$

where

$$
\begin{aligned}
& A(t)=q(t)\left(1-\sum_{i=1}^{n} c_{i}(t)\right)+\sum_{j=1}^{m} \alpha_{j} q_{j}(t)\left(1-\sum_{i=1}^{n} c_{i}\left(t-\sigma_{j}\right)\right), \\
& \tilde{A}(\xi)=\tilde{q}(\xi)\left(1-\sum_{i=1}^{n} \tilde{c}_{i}(\xi)\right)+\sum_{j=1}^{m} \alpha_{j} \tilde{q}_{j}(\xi)\left(1-\sum_{i=1}^{n} \tilde{c}_{i}\left(\xi-\sigma_{j}\right)\right), \\
& \tilde{Q}(\xi, s)=h(\xi, s)-\frac{\tilde{\rho}^{\prime}(\xi)}{\tilde{\rho}(\xi)} \sqrt{H(\xi, s)}, \sigma=\max \left\{\sigma_{1}, \cdots, \sigma_{m}\right\}
\end{aligned}
$$

and

$$
\liminf _{\xi \rightarrow \infty} \int_{\xi+\tau-\sigma^{*}}^{\xi} \frac{1}{\tilde{p}(s)}\left(\int_{s-\sigma^{*}}^{s} \tilde{B}(r) \mathrm{d} r\right) \mathrm{d} s>\frac{1}{\mathrm{e}}
$$

where $\tilde{B}(\xi)=\sum_{j=1}^{m} \frac{\alpha_{j} \tilde{q}_{j}(\xi)}{1+\sum_{i=1}^{n} \widetilde{c}_{i}\left(\xi+\tau-\sigma_{j}\right)}, \tilde{\rho}(\xi)=\rho(t)$ and $\sigma^{*}=\min \left\{\sigma_{1}, \cdots, \sigma_{m}\right\}>\tau$.

Then every solution $u(x, t)$ of $(\mathrm{E}),\left(\mathrm{B}_{1}\right)$ is oscillatory in $G$.

Proof. Suppose that $u(x, t)$ is a non oscillatory solution of $(\mathrm{E}),\left(\mathrm{B}_{1}\right)$, which has no zero in $\Omega \times\left[t_{0}, \infty\right)$ for some $t_{0}>0$. Without loss of generality we may assume that $u(x, t)>0, u\left(x, t-\tau_{i}\right)>0, u\left(x, t-\rho_{k}\right)>0$ and $u\left(x, t-\sigma_{j}\right)>0$, in $\Omega \times[T, \infty), T>t_{0}, k \in I_{s}, j \in I_{m}, i \in I_{n}$, where $T$ is chosen so large that Lemmas 3.1 to 3.4 hold for $t \geq T$. From Lemma 3.1 the function $v(t)$ defined by (1) satisfy the inequality

$$
D_{+}^{\alpha}\left[p(t) D_{+}^{\alpha}\left(v(t)+\sum_{i=1}^{n} c_{i}(t) v\left(t-\tau_{i}\right)\right)\right]+q(t) v(t)+\sum_{j=1}^{m} q_{j}(t) f_{j}\left(v\left(t-\sigma_{j}\right)\right) \leq 0 .
$$

Let $z(t)=v(t)+\sum_{i=1}^{n} c_{i}(t) v\left(t-\tau_{i}\right)$. Then $z(t)$ satisfies either Case (1) or Case (2) of Lemma 3.2.

Case (I): For this case $z(t)>0, D_{+}^{\alpha} z(t)>0$ and $D_{+}^{\alpha}\left[p(t) D_{+}^{\alpha} z(t)\right] \leq 0, t \geq T$. Using Lemma 3.3 and $\left(\mathrm{A}_{5}\right)$, (16) yields

$$
D_{+}^{\alpha}\left[p(t) D_{+}^{\alpha} z(t)\right]+q(t)\left(1-\sum_{i=1}^{n} c_{i}(t)\right) z(t-\sigma)+\left[\sum_{j=1}^{m} \alpha_{j} q_{j}(t)\left(1-\sum_{i=1}^{n} c_{i}\left(t-\sigma_{j}\right)\right)\right] z(t-\sigma) \leq 0, t \geq T .
$$

Define the function $W$ by the generalized Riccati substititution

$$
W(t)=\rho(t) p(t) \frac{D_{+}^{\alpha} z(t)}{z(t-\sigma)} .
$$


then

$$
D_{+}^{\alpha} W(t) \leq D_{+}^{\alpha}(\rho(t)) \frac{W(t)}{\rho(t)}-A(t) \rho(t)-W(t) \frac{D_{+}^{\alpha} z(t-\sigma)}{z(t-\sigma)} .
$$

From $D_{+}^{\alpha}\left(p(t) D_{+}^{\alpha} z(t)\right) \leq 0$ for $t \geq T$ we have $p(t-\sigma) D_{+}^{\alpha} z(t-\sigma) \geq p(t) D_{+}^{\alpha} z(t)$, and consequently by (19) for $t \geq T$, we obtain that

$$
D_{+}^{\alpha}(W(t)) \leq \frac{D_{+}^{\alpha}(\rho(t))}{\rho(t)} W(t)-A(t) \rho(t)-\frac{W^{2}(t)}{\rho(t) p(t-\sigma)}
$$

Let $W(t)=\tilde{W}(\xi)$. Then $D_{+}^{\alpha} W(t)=\tilde{W}^{\prime}(\xi)$ and $D_{+}^{\alpha} \rho(t)=\tilde{\rho}^{\prime}(\xi)$, so the last inequality becomes

$$
\tilde{W}^{\prime}(\xi) \leq \frac{\tilde{\rho}^{\prime}(\xi)}{\tilde{\rho}(\xi)} \tilde{W}(\xi)-\tilde{A}(\xi) \tilde{\rho}(\xi)-\frac{\tilde{W}^{2}(\xi)}{\tilde{\rho}(\xi) \tilde{p}(\xi-\sigma)}
$$

substituting $\xi$ with $s$ multiplying both sides of (21) by $H(\xi, s)$ and integrating from $\xi_{1}$ to $\xi$ for $\xi \geq \xi_{1}$, we have

$$
\begin{aligned}
& \int_{\xi_{1}}^{\xi} H(\xi, s) \tilde{A}(s) \tilde{\rho}(s) \mathrm{d} s \leq \int_{\xi_{1}}^{\xi} H(\xi, s) \frac{\tilde{\rho}^{\prime}(s)}{\tilde{\rho}(s)} \tilde{W}(s) \mathrm{d} s-\int_{\xi_{1}}^{\xi} H(\xi, s) \tilde{W}^{\prime}(s) \mathrm{d} s-\int_{\xi_{1}}^{\xi} H(\xi, s) \frac{\tilde{W}^{2}(s)}{\tilde{\rho}(s) \tilde{p}(s-\sigma)} \mathrm{d} s \\
& \leq H\left(\xi, \xi_{1}\right) \tilde{W}\left(\xi_{1}\right)-\int_{\xi_{1}}^{\xi}\left[-\frac{\partial H}{\partial s}(\xi, s) \tilde{W}(s)-H(\xi, s) \frac{\tilde{\rho}^{\prime}(s)}{\tilde{\rho}(s)} \tilde{W}(s)+\frac{H(\xi, s)}{\tilde{\rho}(s) \tilde{p}(s-\sigma)} \tilde{W}^{\prime}(s)\right] \mathrm{d} s \\
& \leq H\left(\xi, \xi_{1}\right) \tilde{W}\left(\xi_{1}\right)-\int_{\xi_{1}}^{\xi}\left[\sqrt{\left.\frac{H(\xi, s)}{\tilde{\rho}(s) \tilde{p}(s-\sigma)} \tilde{W}(s)+\frac{1}{2} \sqrt{\tilde{\rho}(s) \tilde{p}(s-\sigma)} \tilde{Q}(\xi, s)\right]^{2} \mathrm{~d} s+\int_{\xi_{1}}^{\xi} \frac{\tilde{\rho}(s) \tilde{p}(s-\sigma)}{4} \tilde{Q}^{2}(\xi, s) \mathrm{d} s .}\right.
\end{aligned}
$$

Thus for all $\xi \geq \xi_{1} \geq \xi_{0}$, we conclude that

$$
\int_{\xi_{1}}^{\xi}\left[H(\xi, s) \tilde{A}(s) \tilde{\rho}(s)-\frac{\tilde{\rho}(s) \tilde{p}(s-\sigma)}{4} \tilde{Q}^{2}(\xi, s)\right] \mathrm{d} s \leq H(\xi, \xi) \tilde{W}\left(\xi_{1}\right)
$$

Then, by (22) and $\left(\mathrm{C}_{2}\right)$, for $\xi \geq \xi_{1} \geq \xi_{0}$ we obtain

$$
\int_{\xi_{1}}^{\xi}\left[H(\xi, s) \tilde{A}(s) \tilde{\rho}(s)-\frac{\tilde{\rho}(s) \tilde{p}(s-\sigma)}{4} \tilde{Q}^{2}(\xi, s)\right] \mathrm{d} s \leq H(\xi, \xi)\left|\tilde{W}\left(\xi_{0}\right)\right| \leq H\left(\xi, \xi_{0}\right)\left|\tilde{W}\left(\xi_{0}\right)\right|
$$

Then, by (14) and $\left(\mathrm{C}_{2}\right)$, we have

$$
\begin{gathered}
\int_{\xi_{0}}^{\xi}\left[H(\xi, s) \tilde{A}(s) \tilde{\rho}(s)-\frac{\tilde{\rho}(s) \tilde{p}(s-\sigma)}{4} \tilde{Q}^{2}(\xi, s)\right] \mathrm{d} s \leq H\left(\xi, \xi_{0}\right)\left[\int_{\xi_{0}}^{\xi_{1}} \tilde{A}(s) \tilde{\rho}(s) \mathrm{d} s+\left|\tilde{W}\left(\xi_{0}\right)\right|\right] \\
\limsup _{\xi \rightarrow \infty} \frac{1}{H\left(\xi, \xi_{0}\right)} \int_{\xi_{0}}^{\xi}\left[H(\xi, s) \tilde{A}(s) \tilde{\rho}(s)-\frac{\tilde{\rho}(s) \tilde{p}(s-\sigma)}{4} \tilde{Q}^{2}(\xi, s)\right] \mathrm{d} s \leq \int_{\xi_{0}}^{\xi_{1}} \tilde{A}(s) \tilde{\rho}(s) \mathrm{d} s+\left|\tilde{W}\left(\xi_{0}\right)\right|<\infty,
\end{gathered}
$$

which contradicts (14).

Case (II): Assume that $z(t)$ satisfies (11). Using hypothesis and Lemma 3.3, we have from (16) $t \geq T \geq t_{0}$

$$
D_{+}^{\alpha}\left(p(t) D_{+}^{\alpha} z(t)\right)+\sum_{j=1}^{m}\left[\alpha_{j} \frac{q_{j}(t)}{1+\sum_{i=1}^{n} c_{i}\left(t+\tau-\sigma_{j}\right)}\right] z\left(t+\tau-\sigma^{*}\right) \leq 0 .
$$


Let $z(t)=\tilde{z}(\xi)$. Then $D_{+}^{\alpha} W(t)=\tilde{W}^{\prime}(\xi)$ and $D_{+}^{\alpha} p(t)=\tilde{p}^{\prime}(\xi)$, so the last inequality becomes

$$
\left(\tilde{p}(\xi) \tilde{z}^{\prime}(\xi)\right)^{\prime}+\sum_{j=1}^{m}\left[\alpha_{j} \frac{\tilde{q}_{j}(\xi)}{1+\sum_{i=1}^{n} \tilde{c}_{i}\left(\xi+\tau-\sigma_{j}\right)}\right] \tilde{z}\left(\xi+\tau-\sigma^{*}\right) \leq 0 .
$$

Integrating (26) from $\xi-\sigma^{*}$ to $\xi$ we have

$$
\begin{gathered}
\tilde{p}(\xi) \tilde{z}^{\prime}(\xi)-\tilde{p}\left(\xi-\sigma^{*}\right) \tilde{z}^{\prime}\left(\xi-\sigma^{*}\right)+\int_{\xi-\sigma^{*}}^{\xi} \tilde{B}(s) \tilde{z}\left(\xi+\tau-\sigma^{*}\right) \mathrm{d} s \leq 0, \\
\tilde{z}^{\prime}+\left(\frac{1}{\tilde{p}(\xi)} \int_{\xi-\sigma^{*}}^{\xi} \tilde{B}(s) \mathrm{d} s\right) \tilde{z}\left(\xi+\tau-\sigma^{*}\right) \leq 0 .
\end{gathered}
$$

condition (15) implies that the last inequality has no eventually positive solution, a contradiction. This completes the proof.

Corollary 3.1. Let conditions of Theorem 3.1 be hold. If the inequality (16) has no eventually positive solutions, then every solution $u(x, t)$ of $(\mathrm{E}),\left(\mathrm{B}_{1}\right)$ is oscillatory in $G$.

Corollary 3.2. Let assumption (14) in Theorem 3.1 be replaced by

$$
\lim \sup _{\xi \rightarrow \infty} \frac{1}{H\left(\xi, \xi_{0}\right)} \int_{\xi_{0}}^{\xi} H(\xi, s) \tilde{A}(s) \tilde{\rho}(s) \mathrm{d} s=\infty
$$

and

$$
\lim \sup _{\xi \rightarrow \infty} \frac{1}{H\left(\xi, \xi_{0}\right)} \int_{\xi_{0}}^{\xi} \tilde{p}(s-\sigma) \tilde{\rho}(s) \tilde{Q}^{2}(\xi, s) \mathrm{d} s<\infty .
$$

Then every solution $u(x, t)$ of $(\mathrm{E}),\left(\mathrm{B}_{1}\right)$ is oscillatory in $G$.

Let $H(\xi, s)=(\xi-s)^{n-1},(\xi, s) \in D$ for some integer $n>2$. Then Theorem 3.1, implies the following the result.

Corollary 3.3. Let assumption (14) in Theorem 3.1 be replaced by

$$
\limsup _{\xi \rightarrow \infty}\left(\xi-\xi_{0}\right)^{1-n} \int_{\xi_{0}}^{\xi}\left[(\xi-s)^{n-1} \tilde{A}(s) \tilde{\rho}(s)-\frac{\tilde{\rho}(s) \tilde{p}(s-\sigma)}{4}(\xi-s)^{n-3}\left((n-1)-\frac{\tilde{\rho}^{\prime}(s)}{\tilde{\rho}(s)}(\xi-s)\right)^{2}\right] \mathrm{d} s=\infty,
$$

for some integer $n \geq 3$. Then every solution $u(x, t)$ of $(\mathrm{E}),\left(\mathrm{B}_{1}\right)$ is oscillatory in $G$.

Next we establish conditions for the oscillation of all solutions of $(E),\left(B_{1}\right)$ subject to the following conditions:

$\left.\mathrm{C}_{3}\right) \int_{t_{0}}^{\infty} \frac{\mathrm{d} t}{p(t)}<\infty$

$\left.\mathrm{C}_{4}\right) \frac{f_{j}(u)}{u^{\gamma}} \geq M_{j}>0$ for $u \neq 0$ and $\gamma \in(1, \infty)$ is a ratio of odd integers.

Theorem 3.2. In addition to conditions $\left(\mathrm{C}_{3}\right)$ and $\left(\mathrm{C}_{4}\right)$ assume $\sigma_{j} \geq \tau$ for all $j \in I_{m}$. Then all the solutions of $(\mathrm{E}),\left(\mathrm{B}_{1}\right)$ are oscillatory if

$$
\sum_{j=1}^{m} \int_{\xi_{1}}^{\infty} \tilde{q}_{j}(s)\left(1-\sum_{i=1}^{n} \tilde{c}_{i}\left(s-\sigma_{j}\right)\right)^{\gamma} \tilde{Q}^{\gamma}\left(s-\sigma_{j}\right) \mathrm{d} s=\infty
$$

and 


$$
\sum_{j=1}^{m} \int_{\xi_{1}}^{\infty} \tilde{q}_{j}(s)\left(1-\sum_{i=1}^{n} \tilde{c}_{i}\left(s+\tau-\sigma_{j}\right)\right)^{\gamma} \tilde{Q}^{\gamma}(s) \mathrm{d} s=\infty,
$$

where $\tilde{Q}(\xi)=\int_{\xi}^{\infty} \frac{\mathrm{d} s}{\tilde{p}(s)}$.

Proof. Suppose that $u(x, t)$ is a non oscillatory solution of (E), $\left(\mathrm{B}_{1}\right)$, which has no zero in $\Omega \times\left[t_{0}, \infty\right)$ for some $t_{0}>0$. Without loss of generality we may assume that $u(x, t)>0, u\left(x, t-\tau_{i}\right)>0, u\left(x, t-\rho_{k}\right)>0$ and $u\left(x, t-\sigma_{j}\right)>0$, in $\Omega \times\left[t_{1}, \infty\right), t_{1} \geq t_{0}, k \in I_{s}, j \in I_{m}, i \in I_{n}$. Then the function $v(t)$ defined by (1) satisfies the inequality (16).

Let $z(t)=v(t)+\sum_{i=1}^{n} c_{i}(t) v\left(t-\tau_{i}\right)$. Then $z(t)>0$ for $t \geq t_{1}$. From (16), we have

$$
D_{+}^{\alpha}\left(p(t) D_{+}^{\alpha} z(t)\right) \leq 0 \text { for } t \geq T \geq t_{1}
$$

and $p(t) D_{+}^{\alpha} z(t) \leq p(T) D_{+}^{\alpha} z(T)$ for $t \geq T, D_{+}^{\alpha} z(t) \leq \frac{p(T) D_{+}^{\alpha} z(T)}{p(t)}$.

Let $z(t)=\tilde{z}(\xi)$. Then $D_{+}^{\alpha} z(t)=\tilde{z}^{\prime}(\xi)$, therefore the above inequality becomes $\tilde{z}^{\prime}(\xi) \leq \frac{\tilde{p}\left(\xi_{0}\right) \tilde{z}^{\prime}\left(\xi_{0}\right)}{\tilde{p}(\xi)}$. Integrating the last inequality from $\xi_{0}$ to $\xi$, we have

$$
\tilde{z}(\xi)-\tilde{z}\left(\xi_{0}\right) \leq \tilde{p}\left(\xi_{0}\right) \tilde{z}^{\prime}\left(\xi_{0}\right) \int_{\xi_{0}}^{\xi} \frac{\mathrm{d} s}{\tilde{p}(s)}, \quad \xi \geq \xi_{0} .
$$

since $\tilde{z}(\xi)$ is bounded above. From (30) we obtain

$$
\tilde{z}\left(\xi_{0}\right) \geq-\left(\tilde{p}\left(\xi_{0}\right) \tilde{z}^{\prime}\left(\xi_{0}\right)\right) \int_{\xi_{0}}^{\xi} \frac{\mathrm{d} s}{\tilde{p}(s)}, \quad \xi \geq \xi_{0} .
$$

Letting $\xi \rightarrow \infty$, we obtain

$$
\tilde{z}\left(\xi_{0}\right) \geq-\left(\tilde{p}\left(\xi_{0}\right) \tilde{z}^{\prime}\left(\xi_{0}\right)\right) \tilde{Q}\left(\xi_{0}\right), \quad \xi \geq \xi_{0} .
$$

where $\tilde{Q}(\xi)$ is defined by (28) and $\xi$ is an arbitrary large number.

From Lemma 3.2 there are two possible cases for $z(t)$. First we consider that $z(t)>0, D_{+}^{\alpha} z(t)>0$ for $t \geq T$. Let $z(t)=\tilde{z}(\xi)$. Then $D_{+}^{\alpha} z(t)=\tilde{z}^{\prime}(\xi), q_{j}(t)=\tilde{q}(\xi), v\left(t-\sigma_{j}\right)=\tilde{v}\left(\xi-\sigma_{j}\right)$, using this in (16) we have

$$
\left(\tilde{p}(\xi) \tilde{z}^{\prime}(\xi)\right)^{\prime}+\sum_{j=1}^{m} \tilde{q}_{j}(\xi) \tilde{f}_{j}\left(\tilde{v}\left(\xi-\sigma_{j}\right)\right) \leq 0
$$

Integrating the last inequality from $\xi_{0}$ to $\xi$, we have

$$
\int_{\xi_{0}}^{\xi}\left(\tilde{p}(s) \tilde{z}^{\prime}(s)\right)^{\prime} \mathrm{d} s+\sum_{j=1}^{m} \int_{\xi_{0}}^{\xi} \tilde{q}_{j}(s) \tilde{f}_{j}\left(\tilde{v}\left(s-\sigma_{j}\right)\right) \mathrm{d} s \leq 0 .
$$

By $\left(\mathrm{C}_{4}\right)$ and Lemma 3.3, we have from (32)

$$
\begin{gathered}
\tilde{p}(\xi) \tilde{z}^{\prime}(\xi)-\tilde{p}\left(\xi_{0}\right) \tilde{z}^{\prime}\left(\xi_{0}\right)+\sum_{j=1}^{m} M_{j} \int_{\xi_{0}}^{\xi} \tilde{q}_{j}(s)\left(1-\sum_{i=1}^{n} \tilde{c}_{i}\left(s-\sigma_{j}\right)\right)^{\gamma} \tilde{z}^{\gamma}\left(s-\sigma_{j}\right) \mathrm{d} s \leq 0 . \\
\sum_{j=1}^{m} M_{j} \int_{\xi_{0}}^{\xi} \tilde{q}_{j}(s)\left(1-\sum_{i=1}^{n} \tilde{c}_{i}\left(s-\sigma_{j}\right)\right)^{\gamma} \tilde{z}^{\gamma}\left(s-\sigma_{j}\right) \mathrm{d} s \leq \tilde{p}\left(\xi_{0}\right) \tilde{z}^{\prime}\left(\xi_{0}\right) .
\end{gathered}
$$

Letting $\xi \rightarrow \infty$, we have 


$$
\sum_{j=1}^{m} M_{j} \int_{\xi_{0}}^{\infty} \tilde{q}_{j}(s)\left(1-\sum_{i=1}^{n} \tilde{c}_{i}\left(s-\sigma_{j}\right)\right)^{\gamma} \tilde{z}^{\gamma}\left(s-\sigma_{j}\right) \mathrm{d} s \leq \infty .
$$

For this case $z(t)=\tilde{z}(\xi)$ is increasing, so there exists a number $c$ such that $\tilde{z}(\xi) \geq c>0$ for $\xi \geq \xi_{0}$. Thus there exists a $\xi_{1} \geq \xi_{0}$ such that

$$
\tilde{z}\left(\xi-\sigma_{j}\right) \geq \tilde{Q}\left(\xi-\sigma_{j}\right), \quad \text { for } \xi \geq \xi_{1},
$$

and $j \in I_{m}$, since $\tilde{Q}(\xi) \rightarrow 0$ as $\xi \rightarrow \infty$.

From (34) and (35) we have

$$
\sum_{j=1}^{m} M_{j} \int_{\xi_{0}}^{\infty} \tilde{q}_{j}(s)\left(1-\sum_{i=1}^{n} \tilde{c}_{i}\left(s-\sigma_{j}\right)\right)^{\gamma} \tilde{Q}^{\gamma}\left(s-\sigma_{j}\right) \mathrm{d} s<\infty
$$

which contradicts (27).

Next we consider the case that $z(t)>0$ and $D_{+}^{\alpha} z(t)<0$ for $t \geq T$. From (31), we have

$$
\tilde{z}(\xi) \geq-\left(\tilde{p}(\xi) \tilde{z}^{\prime}(\xi)\right) \tilde{Q}(\xi) \text { for } \xi \geq \xi_{0} .
$$

Consider $D_{+}^{\alpha}\left(p(t) D_{+}^{\alpha} z(t)\right)^{-\gamma+1}$, since $\gamma$ is an odd ratio integer.

$$
D_{+}^{\alpha}\left(p(t) D_{+}^{\alpha} z(t)\right)^{-\gamma+1}=(-\gamma+1)\left(p(t) D_{+}^{\alpha} z(t)\right)^{-\gamma} D_{+}^{\alpha}\left(p(t) D_{+}^{\alpha} z(t)\right) .
$$

Let $z(t)=\tilde{z}(\xi)$. Then $D_{+}^{\alpha} z(t)=\tilde{z}^{\prime}(\xi)$

$$
\begin{aligned}
& \left(\left(\tilde{p}(\xi) \tilde{z}^{\prime}(\xi)\right)^{-\gamma+1}\right)^{\prime}=(-\gamma+1)\left(\tilde{p}(\xi) \tilde{z}^{\prime}(\xi)\right)^{-\gamma}\left(\tilde{p}(\xi) \tilde{z}^{\prime}(\xi)\right)^{\prime} \\
& \leq(-\gamma+1)\left(\tilde{p}(\xi) \tilde{z}^{\prime}(\xi)\right)^{-\gamma}\left(-\sum_{j=1}^{m} \tilde{q}_{j}(\xi) \tilde{f}_{j}\left(\tilde{v}\left(\xi-\sigma_{j}\right)\right)\right) \\
& \leq(-\gamma+1)\left(\tilde{p}(\xi) \tilde{z}^{\prime}(\xi)\right)^{-\gamma}\left(-\sum_{j=1}^{m} M_{j} \tilde{q}_{j}(\xi) \tilde{v}^{\gamma}\left(\xi-\sigma_{j}\right)\right) \\
& \leq(-\gamma+1)\left(\tilde{p}(\xi) \tilde{z}^{\prime}(\xi)\right)^{-\gamma}\left(-\sum_{j=1}^{m} M_{j} \tilde{q}_{j}(\xi)\left(1-\sum_{i=1}^{n} \tilde{c}_{i}\left(\xi+\tau-\sigma_{j}\right)\right)^{\gamma} \tilde{z}^{\gamma}(\xi)\right) \\
& \leq-(\gamma-1) \sum_{j=1}^{m} M_{j} \tilde{q}_{j}(\xi)\left(1-\sum_{i=1}^{n} \tilde{c}_{i}\left(\xi+\tau-\sigma_{j}\right)\right)^{\gamma} \tilde{Q}^{\gamma}(\xi)
\end{aligned}
$$

here we have used $\left(\mathrm{C}_{4}\right)$, (37) and Lemma 3.4. Integrating the last inequality from $\xi_{0}$ to $\xi$, we obtain

$$
(\gamma-1) \sum_{j=1}^{m} M_{j} \int_{\xi_{0}}^{\xi} \tilde{q}_{j}(s)\left(1-\sum_{i=1}^{n} \tilde{c}_{i}\left(s+\tau-\sigma_{j}\right)\right)^{\gamma} \tilde{Q}^{\gamma}(s) \mathrm{d} s \leq\left(\tilde{p}\left(\xi_{0}\right) \tilde{z}^{\prime}\left(\xi_{0}\right)\right)^{-\gamma+1}
$$

and so letting $\xi \rightarrow \infty$, we obtain

$$
\sum_{j=1}^{m} M_{j} \int_{\xi_{0}}^{\infty} \tilde{q}_{j}(s)\left(1-\sum_{i=1}^{n} \tilde{c}_{i}\left(s+\tau-\sigma_{j}\right)\right)^{\gamma} \tilde{Q}^{\gamma}(s) \mathrm{d} s<\infty
$$

which contradicts (28). This completes the proof.

Next we consider $(E),\left(B_{1}\right)$ subject to the following conditions:

C) $\frac{f_{j}(u)}{u^{\gamma}} \geq M_{j}>0$ for $u \neq 0$ and $\gamma \in(0,1)$ is a ratio of odd positive integers.

Theorem 3.3. In addition to conditions $\left(\mathrm{C}_{3}\right)$ and $\left(\mathrm{C}_{5}\right)$ assume that 


$$
\int_{\xi_{0}}^{\infty} \sum_{j=1}^{m} \tilde{q}_{j}(s)\left(1-\sum_{i=1}^{n} \tilde{c}_{i}\left(s-\sigma_{j}\right)\right)^{\gamma} \tilde{Q}\left(s-\sigma_{j}\right) \mathrm{d} s=\infty
$$

and

$$
\int_{\xi_{1}}^{\infty} \sum_{j=1}^{m} \tilde{q}_{j}(s)\left(1-\sum_{i=1}^{n} \tilde{c}_{i}\left(s+\tau-\sigma_{j}\right)\right)^{\gamma} \tilde{Q}(s) \mathrm{d} s=\infty .
$$

Then every solution $u(x, t)$ of $(\mathrm{E}),\left(\mathrm{B}_{1}\right)$ is oscillatory in $G$.

Proof. Without loss of generality we may assume that $u(x, t)>0, u\left(x, t-\tau_{i}\right)>0, u\left(x, t-\rho_{k}\right)>0$ and $u\left(x, t-\sigma_{j}\right)>0$, in $\Omega \times\left[t_{1}, \infty\right), t_{1} \geq t_{0}, k \in I_{s}, j \in I_{i}, j \in I_{n}$, is a solution of (E), ( $\left.\mathrm{B}_{1}\right)$. Therefore $D_{+}^{\alpha}\left(p(t) D_{+}^{\alpha} z(t)\right) \leq 0$ for $t \geq T \geq t_{1}$.

If $D_{+}^{\alpha} z(t)>0$ for $t \geq T$, we have from (34) and (36). For large $t$ we have $Q(t)=\tilde{Q}(\xi) \leq 1$ and $\tilde{Q}^{\gamma}(\xi) \leq \tilde{Q}(\xi)$. Therefore from (36), we obtain

$$
\int_{\xi_{1}}^{\infty} \sum_{j=1}^{m} M_{j} \tilde{q}_{j}(s)\left(1-\sum_{i=1}^{n} \tilde{c}_{i}\left(s-\sigma_{j}\right)\right)^{\gamma} \tilde{Q}\left(s-\sigma_{j}\right) \mathrm{d} s<\infty,
$$

which contradicts (38). For this case $D_{+}^{\alpha} z(t)<0$ for $t \geq T$, from (33)

$$
\begin{aligned}
& \tilde{p}(\xi) \tilde{z}^{\prime}(\xi)-\tilde{p}\left(\xi_{0}\right) \tilde{z}^{\prime}\left(\xi_{0}\right)+\int_{\xi_{0}}^{\xi} \sum_{j=1}^{m} M_{j} \tilde{q}_{j}(s)\left(1-\sum_{i=1}^{n} \tilde{c}_{i}\left(s+\tau-\sigma_{j}\right)\right)^{\gamma} \tilde{z}^{\gamma}\left(s+\tau-\sigma_{j}\right) \mathrm{d} s \leq 0, \text { for } \xi \geq \xi_{0} . \\
& -\tilde{z}^{\prime}(\xi) \geq \frac{1}{\tilde{p}(\xi)} \int_{\xi_{0}}^{\xi} \sum_{j=1}^{m} M_{j} \tilde{q}_{j}(s)\left(1-\sum_{i=1}^{n} \tilde{c}_{i}\left(s+\tau-\sigma_{j}\right)\right)^{\gamma} \tilde{z}^{\gamma}\left(s+\tau-\sigma_{j}\right) \mathrm{d} s \text { for } \xi \geq \xi_{0} .
\end{aligned}
$$

We consider the fractional differential $D_{+}^{\alpha} z^{2 \varepsilon}(t)$ where $\varepsilon>0$ such that $2 \varepsilon<1-\gamma$

$$
-D_{+}^{\alpha} z^{2 \varepsilon}(t)=-2 \varepsilon z^{2 \varepsilon-1}(t) D_{+}^{\alpha} z(t) .
$$

Let $z(t)=\tilde{z}(\xi)$. Then $D_{+}^{\alpha} z(t)=\tilde{z}^{\prime}(\xi)$.

$$
\begin{aligned}
-\left(\tilde{z}^{2 \varepsilon}(\xi)\right)^{\prime} & =-2 \varepsilon\left(\tilde{z}^{2 \varepsilon-1}(\xi) \tilde{z}^{\prime}(\xi)\right) \\
& \geq 2 \varepsilon\left(\tilde{z}^{2 \varepsilon-1}(\xi)\right) \frac{1}{\tilde{p}(\xi)} \int_{\xi_{0}}^{\xi} \sum_{j=1}^{m} M_{j} \tilde{q}_{j}(s)\left(1-\sum_{i=1}^{n} \tilde{c}_{i}\left(s+\tau-\sigma_{j}\right)\right)^{\gamma} \tilde{z}^{\gamma}\left(s+\tau-\sigma_{j}\right) \mathrm{d} s \\
& \geq 2 \varepsilon \frac{1}{\tilde{p}(\xi)} \int_{\xi_{0}}^{\xi} \sum_{j=1}^{m} M_{j} \tilde{q}_{j}(s)\left(1-\sum_{i=1}^{n} \tilde{c}_{i}\left(s+\tau-\sigma_{j}\right)\right)^{\gamma} \tilde{z}^{2 \varepsilon+\gamma-1}\left(s+\tau-\sigma_{j}\right) \mathrm{d} s \\
& \geq 2 \varepsilon \frac{1}{\tilde{p}(\xi)} \int_{\xi_{0}}^{\xi} \sum_{j=1}^{m} M_{j} \tilde{q}_{j}(s)\left(1-\sum_{i=1}^{n} \tilde{c}_{i}\left(s+\tau-\sigma_{j}\right)\right)^{\gamma} \tilde{z}^{2 \varepsilon+\gamma-1}(s) \mathrm{d} s
\end{aligned}
$$

according as $\tau \geq \sigma_{j}$ or $\tau \leq \sigma_{j}$ and $\tilde{z}(\xi)$ is decreasing. Since $c_{1} \geq \tilde{z}(\xi)>0$ for $\xi \geq \xi_{0}$ where $c_{1}>0$ is a constant, there exist positive number $k$ such that

$$
-\left(\tilde{z}^{2 \varepsilon}(\xi)\right)^{\prime} \geq \frac{k}{\tilde{p}(\xi)} \int_{\xi_{0}}^{\xi} \sum_{j=1}^{m} M_{j} \tilde{q}_{j}(s)\left(1-\sum_{i=1}^{n} \tilde{c}_{i}\left(s+\tau-\sigma_{j}\right)\right)^{\gamma} \mathrm{d} s .
$$

Integrating and rearranging we obtain

$$
\tilde{z}^{2 \varepsilon}\left(\xi_{0}\right)-\tilde{z}^{2 \varepsilon}(\xi) \geq \int_{\xi_{0}}^{\xi} \sum_{j=1}^{m} M_{j} \tilde{q}_{j}(s)\left(1-\sum_{i=1}^{n} \tilde{c}_{i}\left(s+\tau-\sigma_{j}\right)\right)^{\gamma}\left(\int_{\xi_{0}}^{\xi} \frac{\mathrm{d} r}{\tilde{p}(r)}\right) \mathrm{d} s,
$$


and so letting $\xi \rightarrow \infty$, we have

$$
\int_{\xi_{0}}^{\infty} \sum_{j=1}^{m} M_{j} \tilde{q}_{j}(s)\left(1-\sum_{i=1}^{n} \tilde{c}_{i}\left(s+\tau-\sigma_{j}\right)\right)^{\gamma} \tilde{Q}(s) \mathrm{d} s<\infty
$$

which contradicts (39). This completes the proof.

\section{Oscillation of (E), ( $\left.\mathrm{B}_{2}\right)$}

In this section we establish sufficient conditions for the oscillation of all solutions of $(E),\left(B_{2}\right)$. For this we need the following:

The smallest eigen value $\beta_{0}$ of the Dirichlet problem

$$
\begin{gathered}
\Delta \omega(x)+\beta \omega(x)=0 \text { in } \Omega \\
\omega(x)=0 \text { on } \partial \Omega,
\end{gathered}
$$

is positive and the corresponding eigen function $\phi(x)$ is positive in $\Omega$.

Theorem 4.1. Let all the conditions of Theorem 3.1 be hold. Then every solution of $(E),\left(B_{2}\right)$ oscillates in $G$. Proof. Suppose that $u(x, t)$ is a non oscillatory solution of $(\mathrm{E}),\left(\mathrm{B}_{2}\right)$, which has no zero in $\Omega \times\left[t_{0}, \infty\right)$ for some $t_{0}>0$. Without loss of generality, we may assume that $u(x, t)>0, u\left(x, t-\tau_{i}\right)>0, u\left(x, t-\rho_{k}\right)>0$ and $u\left(x, t-\sigma_{j}\right)>0$ in $\Omega \times\left[t_{1}, \infty\right), t_{1} \geq t_{0}, k \in I_{s}, j \in I_{m}, i \in I_{n}$. Multiplying both sides of the Equation (E) by $\phi(x)>0$ and integrating with respect to $x$ over $\Omega$.

We obtain for $t \geq t_{1}$,

$$
\begin{aligned}
& \int_{\Omega} D_{+, t}^{\alpha}\left[p(t) D_{+, t}^{\alpha}\left(u(x, t)+\sum_{i=1}^{n} c_{i}(t) u\left(x, t-\tau_{i}\right)\right)\right] \phi(x) \mathrm{d} x+\int_{\Omega} q(x, t) u(x, t) \phi(x) \mathrm{d} x \\
& +\sum_{j=1}^{m} \int_{\Omega} q_{j}(x, t) f_{j}\left(u\left(x, t-\sigma_{j}\right)\right) \phi(x) \mathrm{d} x \\
& =a(t) \int_{\Omega} \Delta u(x, t) \phi(x) \mathrm{d} x+\sum_{k=1}^{s} a_{k}(t) \int_{\Omega} \Delta u\left(x, t-\rho_{k}\right) \phi(x) \mathrm{d} x+\int_{\Omega} F(x, t) \phi(x) \mathrm{d} x .
\end{aligned}
$$

Using Green's formula and boundary condition $\left(\mathrm{B}_{2}\right)$ it follows that

$$
\int_{\Omega} \Delta u(x, t) \phi(x) \mathrm{d} x=\int_{\Omega} u(x, t) \Delta \phi(x) \mathrm{d} x=-\beta_{0} \int_{\Omega} u(x, t) \phi(x) \mathrm{d} x \leq 0, \quad t \geq t_{1},
$$

and for $k \in I_{s}$.

$$
\int_{\Omega} \Delta u\left(x, t-\rho_{k}\right) \phi(x) \mathrm{d} x=\int_{\Omega} u\left(x, t-\rho_{k}\right) \Delta \phi(x) \mathrm{d} x=-\beta_{0} \int_{\Omega} u\left(x, t-\rho_{k}\right) \phi(x) \mathrm{d} x \leq 0, \quad t \geq t_{1},
$$

Also from $\left(A_{3}\right),\left(A_{5}\right)$, we obtain

$$
\int_{\Omega} q(x, t) u(x, t) \phi(x) \mathrm{d} x \geq q(t) \int_{\Omega} u(x, t) \phi(x) \mathrm{d} x, \quad t \geq t_{1}
$$

and using and Jensen's inequality we get

$$
\begin{aligned}
& \int_{\Omega} q_{j}(x, t) f_{j}\left(u\left(x, t-\sigma_{j}\right)\right) \phi(x) \mathrm{d} x \geq q_{j}(t) \int_{\Omega} f_{j}\left(u\left(x, t-\sigma_{j}\right)\right) \phi(x) \mathrm{d} x \\
& \geq q_{j}(t) \int_{\Omega} \phi(x) \mathrm{d} x f_{j}\left(\int_{\Omega} u\left(x, t-\sigma_{j}\right) \phi(x) \mathrm{d} x\right)\left(\int_{\Omega} \phi(x) \mathrm{d} x\right)^{-1}, t \geq t_{1} \text { and } j \in I_{m} .
\end{aligned}
$$

Set

$$
v(t)=\int_{\Omega} u(x, t) \phi(x) \mathrm{d} x\left(\int_{\Omega} \phi(x) \mathrm{d} x\right)^{-1}, t \geq t_{1} .
$$

In view of (41)-(45) and ( $\left.\mathrm{A}_{6}\right)$, (40) yield 


$$
D_{+}^{\alpha}\left[p(t) D_{+}^{\alpha}\left[v(t)+\sum_{i=1}^{n} c_{i}(t) v\left(t-\tau_{i}\right)\right]\right]+q(t) v(t)+\sum_{j=1}^{m} q_{j}(t) f_{j}\left(v\left(t-\sigma_{j}\right)\right) \leq 0 .
$$

for $t \geq t_{1}$. Rest of the proof is similar to that of Theorems 3.1 and hence the details are omitted.

Using the above theorem, we derive the following Corollaries.

Corollary 4.1. If the inequality (46) has no eventually positive solutions, then every solution $u(x, t)$ of $(\mathrm{E})$, $\left(\mathrm{B}_{2}\right)$ is oscillatory in $\mathrm{G}$.

Corollary 4.2. Let the conditions of Corollary 3.2 hold; then every solution $u(x, t)$ of $(\mathrm{E}),\left(\mathrm{B}_{2}\right)$ is oscillatory in G.

Corollary 4.3. Let the conditions of Corollary 3.3 hold; then every solution $u(x, t)$ of $(E),\left(B_{2}\right)$ is oscillatory in G.

Theorem 4.2. Let the conditions of Theorem 3.2 hold; then every solution $u(x, t)$ of $(E),\left(B_{2}\right)$ is oscillatory in $\mathrm{G}$.

Theorem 4.3. Let the conditions of Theorem 3.3 hold; then every solution $u(x, t)$ of $(\mathrm{E}),\left(\mathrm{B}_{2}\right)$ is oscillatory in G.

The proof Theorems 4.2 and 4.3 are similar to that of Theorem 4.1 and ends details are omitted.

\section{Examples}

In this section we give some examples to illustrate our results established in Sections 3 and 4.

Example 1. Consider the fractional neutral partial differential equation

$$
\begin{aligned}
& D_{+, t}^{\frac{1}{2}}\left(t^{\frac{7}{4}} D_{+, t}^{\frac{1}{2}}\left(u(x, t)+\frac{1}{t^{\frac{1}{4}}} u(x, t-2 \pi)\right)-\left(\frac{\pi}{\left(\Gamma\left(\frac{1}{4}\right)\right)^{2}}+\frac{3}{4} \sqrt{\frac{\pi}{2}}\right) t u(x, t)\right. \\
& -\left(\frac{21}{5} \frac{\pi}{\left(\Gamma\left(\frac{1}{4}\right)\right)^{2}} t^{\frac{5}{4}}+\frac{2 \sqrt{2 \pi}}{\left(\Gamma\left(\frac{1}{4}\right)\right)^{2}} t^{\frac{1}{2}}\right) u(x, t-4 \pi) \\
& =\left(\frac{21}{5} \frac{\pi}{\left(\Gamma\left(\frac{1}{4}\right)\right)^{2}} t^{\frac{5}{4}}+t^{\frac{7}{4}}+\frac{\pi}{\left(\Gamma\left(\frac{1}{4}\right)\right)^{2}} t+\frac{3}{4} \sqrt{\frac{\pi}{2}} t+t^{\frac{3}{2}}\right) \Delta u\left(x, t-\frac{\pi}{2}\right)
\end{aligned}
$$

for $(x, t) \in(0, \pi) \times[0, \infty)$, with the boundary condition

$$
u(0, t)=u(\pi, t)=0, t \geq 0 .
$$

Example 1 is particular case of Equation (E). Here

$$
\begin{aligned}
& \alpha=1 / 2, N=1, n=1, s=1, m=1, p(t)=t^{\frac{7}{4}}, c_{1}(t)=\frac{1}{t^{\frac{1}{4}}}, \tau_{1}=2 \pi, \tau=\max \left\{\tau_{1}\right\}=2 \pi, \\
& q(x, t)=-\left(\frac{\pi}{\left(\Gamma\left(\frac{1}{4}\right)\right)^{2}}+\frac{3}{4} \sqrt{\frac{\pi}{2}}\right) t, \quad q_{1}(x, t)=-\left(\frac{21}{5} \frac{\pi}{\left(\Gamma\left(\frac{1}{4}\right)\right)^{2}} t^{\frac{5}{4}}+\frac{2 \sqrt{2 \pi}}{\left(\Gamma\left(\frac{1}{4}\right)\right)^{2}} t^{\frac{1}{2}}\right), \sigma_{1}=4 \pi, \sigma=\max \left\{\sigma_{1}\right\}=4 \pi,
\end{aligned}
$$




$$
a_{1}(t)=\left(\frac{21}{5} \frac{\pi}{\left(\Gamma\left(\frac{1}{4}\right)\right)^{2}} t^{\frac{5}{4}}+t^{\frac{7}{4}}+\frac{\pi}{\left(\Gamma\left(\frac{1}{4}\right)\right)^{2}} t+\frac{3}{4} \sqrt{\frac{\pi}{2}} t+t^{\frac{3}{2}}\right), \quad \rho_{1}=\frac{\pi}{2}
$$

and $f_{1}(u)=u$.

It is easy to see that

$$
\begin{aligned}
& q(t)=\min _{x \in \bar{\Omega}} q(x, t)=-\left(\frac{\pi}{\left(\Gamma\left(\frac{1}{4}\right)\right)^{2}}+\frac{3}{4} \sqrt{\frac{\pi}{2}}\right) t, \\
& q_{1}(t)=\min _{x \in \bar{\Omega}} q_{1}(x, t)=-\left(\frac{21}{5} \frac{\pi}{\left(\Gamma\left(\frac{1}{4}\right)\right)^{2}} t^{\frac{5}{4}}+\frac{2 \sqrt{2 \pi}}{\left(\Gamma\left(\frac{1}{4}\right)\right)^{2}} t^{\frac{1}{2}}\right), \\
& \tilde{A}(\xi)=\tilde{q}(\xi)\left(1-\sum_{i=1}^{n} \tilde{c}_{i}(\xi)\right)+\sum_{j=1}^{m} \alpha_{j} \tilde{q}_{j}(\xi)\left(1-\sum_{i=1}^{n} \tilde{c}_{i}\left(\xi-\sigma_{j}\right)\right),
\end{aligned}
$$

Here $n=1, m=1$, so we have

$$
\tilde{A}(\xi)=\tilde{q}(\xi)\left(1-\tilde{c}_{1}(\xi)\right)+\alpha_{1} \tilde{q}_{1}(\xi)\left(1-\tilde{c}_{1}\left(\xi-\sigma_{1}\right)\right)
$$

Take $\alpha_{1}=1$

$$
\begin{gathered}
\tilde{A}(\xi)=-\left(\frac{\pi}{\left(\Gamma\left(\frac{1}{4}\right)\right)^{2}}+\frac{3}{4} \sqrt{\frac{\pi}{2}}\right) \xi\left(1-\frac{1}{\xi^{\frac{1}{4}}}\right)-\left(\frac{21}{5} \frac{\pi}{\left(\Gamma\left(\frac{1}{4}\right)\right)^{2}} \xi^{\frac{5}{4}}+\frac{2 \sqrt{2 \pi}}{\left(\Gamma\left(\frac{1}{4}\right)\right)^{2}} \xi^{\frac{1}{2}}\right)\left(1-\frac{1}{(\xi-4 \pi)^{\frac{1}{4}}}\right), \\
\tilde{B}(\xi)=\sum_{j=1}^{m} \frac{\alpha_{j} \tilde{q}_{j}(\xi)}{1+\sum_{i=1}^{n} \tilde{c}_{i}\left(\xi+\tau-\sigma_{j}\right)}
\end{gathered}
$$

Here $m=1, n=1$ so we have

$$
\begin{gathered}
\tilde{B}(\xi)=\frac{\alpha_{1} \tilde{q}_{1}(\xi)}{1+\tilde{c}_{1}\left(\xi+\tau-\sigma_{1}\right)} \\
\tilde{B}(\xi)=-\left(\frac{21}{5} \frac{\pi}{\left(\Gamma\left(\frac{1}{4}\right)\right)^{2}} \xi^{\frac{5}{4}}+\frac{2 \sqrt{2 \pi}}{\left(\Gamma\left(\frac{1}{4}\right)\right)^{2}} \xi^{\frac{1}{2}}\right) \frac{(\xi-2 \pi)^{\frac{1}{4}}}{(\xi-2 \pi)^{\frac{1}{4}}+1}
\end{gathered}
$$

Consider

$$
\int_{\xi_{0}}^{\xi}\left[(\xi-s)^{n-1} \tilde{A}(s) \tilde{\rho}(s)-\frac{\tilde{\rho}(s) \tilde{p}(s-\sigma)}{4}(\xi-s)^{n-3}\left((n-1)-\frac{\tilde{\rho}^{\prime}(s)}{\tilde{\rho}(s)}(\xi-s)\right)^{2}\right] \mathrm{d} s
$$


Choose $n=3$ and $\tilde{\rho}(\xi)=1$, we get

$$
\begin{aligned}
& \int_{\xi_{0}}^{\xi}\left[(\xi-s)^{2} \tilde{A}(s) \tilde{\rho}(s)-\tilde{\rho}(s) \tilde{p}(s-\sigma)\right] \mathrm{d} s \\
& =\int_{\xi_{0}}^{\xi}\left[(\xi-s)^{2}\left\{-\left(\frac{\pi}{\left(\Gamma\left(\frac{1}{4}\right)\right)^{2}}+\frac{3}{4} \sqrt{\frac{\pi}{2}}\right) s\left(1-\frac{1}{s^{\frac{1}{4}}}\right)-\left(\frac{21}{5} \frac{\pi}{\left(\Gamma\left(\frac{1}{4}\right)\right)^{2}} s^{\frac{5}{4}}+\frac{2 \sqrt{2 \pi}}{\left(\Gamma\left(\frac{1}{4}\right)\right)^{2}} s^{\frac{1}{2}}\right)\left(1-\frac{1}{(s-4 \pi)^{\frac{1}{4}}}\right)\right\}-(s-4 \pi)^{\frac{7}{4}}\right] \mathrm{d} s \\
& <\int_{\xi_{0}}^{\xi}\left[s^{2}\left\{-\left(\frac{\pi}{\left(\Gamma\left(\frac{1}{4}\right)\right)^{2}}+\frac{3}{4} \sqrt{\frac{\pi}{2}}\right) s-\left(\frac{21}{5} \frac{\pi}{\left(\Gamma\left(\frac{1}{4}\right)\right)^{2}} s^{\frac{5}{4}}+\frac{2 \sqrt{2 \pi}}{\left(\Gamma\left(\frac{1}{4}\right)\right)^{2}} s^{\frac{1}{2}}\right)\right\}-(s-4 \pi)^{\frac{7}{4}}\right] \mathrm{d} s \rightarrow \infty, \text { as } \xi \rightarrow \infty .
\end{aligned}
$$

Thus all the conditions of Corollary 3.3 are satisfied. Hence every solution of $\left(E_{1}\right)$, (47) oscillates in $(0, \pi) \times[0, \infty)$. In fact $u(x, t)=\sin x \cos t$ is such a solution.

Example 2. Consider the fractional neutral partial differential equation

$$
\begin{aligned}
& D_{+, t}^{\frac{1}{2}}\left(t^{\frac{5}{4}} D_{+, t}^{\frac{1}{2}}\left(u(x, t)+\frac{1}{t^{\frac{1}{4}}} u(x, t-2 \pi)\right)+\left(\frac{5}{24 \pi}\left(\Gamma\left(\frac{1}{4}\right)\right)^{2} t^{\frac{3}{4}}+t^{\frac{5}{4}}+\frac{\pi}{\left(\Gamma\left(\frac{1}{4}\right)\right)^{2}} t^{\frac{1}{2}}+\sqrt{\frac{2}{\pi}} t^{\frac{1}{2}}+t\right) u\left(x, t-\frac{5 \pi}{2}\right)\right. \\
& =-\left(\frac{5}{24 \pi}\left(\Gamma\left(\frac{1}{4}\right)\right)^{2} t^{\frac{3}{4}}+\frac{\pi}{\left(\Gamma\left(\frac{1}{4}\right)\right)^{2}} t^{\frac{1}{2}}+\sqrt{\frac{2}{\pi}} t^{\frac{1}{2}}\right) \Delta u(x, t)+\frac{\pi}{\left(\Gamma\left(\frac{1}{4}\right)\right)^{2}} \sqrt{\frac{\pi}{2}} \cos x \sin t
\end{aligned}
$$

for $(x, t) \in(0, \pi) \times[0, \infty)$, with the boundary condition

$$
u_{x}(0, t)=u_{x}(\pi, t)=0, \quad t \geq 0
$$

Here

$$
\begin{gathered}
\alpha=1 / 2, N=1, n=1, s=1, m=1, p(t)=t^{\frac{5}{4}}, c_{1}(t)=\frac{1}{t^{\frac{1}{4}}}, \tau_{1}=2 \pi \\
\tau=\max \left\{\tau_{1}\right\}=2 \pi, q(x, t)=0, q_{1}(x, t)=\left(\frac{5}{24 \pi}\left(\Gamma\left(\frac{1}{4}\right)\right)^{2} t^{\frac{3}{4}}+t^{\frac{5}{4}}+\frac{\pi}{\left(\Gamma\left(\frac{1}{4}\right)\right)^{2}} t^{\frac{1}{2}}+\sqrt{\frac{2}{\pi}} t^{\frac{1}{2}}+t\right), \sigma_{1}=5 \pi / 2, \\
\sigma=\max \left\{\sigma_{1}\right\}=\frac{5 \pi}{2}, a_{1}(t)=-\left(\frac{5}{24 \pi}\left(\Gamma\left(\frac{1}{4}\right)\right)^{2} t^{\frac{3}{4}}+\frac{\pi}{\left(\Gamma\left(\frac{1}{4}\right)\right)^{2}} t^{\frac{1}{2}}+\sqrt{\frac{2}{\pi}} t^{\frac{1}{2}}\right), \rho_{1}=0,
\end{gathered}
$$




$$
F(x, t)=\frac{\pi}{\left(\Gamma\left(\frac{1}{4}\right)\right)^{2}} \sqrt{\frac{\pi}{2}} \cos x \sin t \text { and } f_{1}(u)=u
$$

It is easy to see that

$$
q(t)=0, \quad q_{1}(t)=\left(\frac{5}{24 \pi}\left(\Gamma\left(\frac{1}{4}\right)\right)^{2} t^{\frac{3}{4}}+t^{\frac{5}{4}}+\frac{\pi}{\left(\Gamma\left(\frac{1}{4}\right)\right)^{2}} t^{\frac{1}{2}}+\sqrt{\frac{2}{\pi}} t^{\frac{1}{2}}+t\right)
$$

Take $\alpha_{1}=1$

$$
\begin{gathered}
\tilde{A}(\xi)=\left(\frac{5}{24 \pi}\left(\Gamma\left(\frac{1}{4}\right)\right)^{2} \xi^{\frac{3}{4}}+\xi^{\frac{5}{4}}+\frac{\pi}{\left(\Gamma\left(\frac{1}{4}\right)\right)^{2}} \xi^{\frac{1}{2}}+\sqrt{\frac{2}{\pi}} \xi^{\frac{1}{2}}+\xi\right)\left(1-\frac{1}{\left(\xi-\frac{5 \pi}{2}\right)^{\frac{1}{4}}}\right) \\
\tilde{B}(\xi)=\left(\frac{5}{24 \pi}\left(\Gamma\left(\frac{1}{4}\right)\right)^{2} \xi^{\frac{3}{4}}+\xi^{\frac{5}{4}}+\frac{\pi}{\left(\Gamma\left(\frac{1}{4}\right)\right)^{2}} \xi^{\frac{1}{2}}+\sqrt{\frac{2}{\pi}} \xi^{\frac{1}{2}}+\xi\right) \frac{\left(\xi-\frac{\pi}{2}\right)^{\frac{1}{4}}}{1+\left(\xi-\frac{\pi}{2}\right)^{\frac{1}{4}}}
\end{gathered}
$$

Consider $\int_{\xi_{0}}^{\xi}\left[(\xi-s)^{n-1} \tilde{A}(s) \tilde{\rho}(s)-\frac{\tilde{\rho}(s) \tilde{p}(s-\sigma)}{4}(\xi-s)^{n-3}\left((n-1)-\frac{\tilde{\rho}^{\prime}(s)}{\tilde{\rho}(s)}(\xi-s)\right)^{2}\right] \mathrm{d} s$

Choose $n=3$ and $\tilde{\rho}(\xi)=1$, we get

$$
\begin{aligned}
& \int_{\xi_{0}}^{\xi}\left[(\xi-s)^{2} \tilde{A}(s) \tilde{\rho}(s)-\tilde{\rho}(s) \tilde{p}(s-\sigma)\right] \mathrm{d} s \\
& =\int_{\xi_{0}}^{\xi}\left[(\xi-s)^{2}\left(\frac{5}{24 \pi}\left(\Gamma\left(\frac{1}{4}\right)\right)^{2} s^{\frac{3}{4}}+s^{\frac{5}{4}}+\frac{\pi}{\left(\Gamma\left(\frac{1}{4}\right)\right)^{2}} s^{\frac{1}{2}}+\sqrt{\frac{2}{\pi}} s^{\frac{1}{2}}+s\right)\left(1-\frac{1}{\left(s-\frac{5 \pi}{2}\right)^{\frac{1}{4}}}\right)-\left(s-\frac{5 \pi}{2}\right)^{\frac{5}{4}}\right] \mathrm{d} s \\
& <\int_{\xi_{0}}^{\xi}\left[s^{2}\left[\frac{5}{24 \pi}\left(\Gamma\left(\frac{1}{4}\right)\right)^{2} s^{\frac{3}{4}}+s^{\frac{5}{4}}+\frac{\pi}{\left(\Gamma\left(\frac{1}{4}\right)\right)^{2}} s^{\frac{1}{2}}+\sqrt{\frac{2}{\pi}} s^{\frac{1}{2}}+s\right)-\left(s-\frac{5 \pi}{2}\right)^{\frac{5}{4}}\right] \mathrm{d} s \rightarrow \infty, \text { as } \xi \rightarrow \infty .
\end{aligned}
$$

Thus all the conditions of Corollary 3.3 are satisfied. Therefore every solution of $\left(E_{2}\right),(48)$ oscillates in $(0, \pi) \times[0, \infty)$. In fact $u(x, t)=\cos x \sin t$ is such a solution.

\section{Acknowledgements}

The authors thank Prof. E. Thandapani for his support to complete the paper. Also the authors express their sincere thanks to the referee for valuable suggestions. 


\section{References}

[1] Miller, K.S. and Ross, B. (1993) An Introduction to the Fractional Calculus and Fractional Differential Equations. John Wiley and Sons, New York.

[2] Podlubny, I. (1999) Fractional Differential Equations. Academic Press, San Diego.

[3] Kilbas, A.A., Srivastava, H.M. and Trujillo, J.J. (2006) Theory and Applications of Fractional Differential Equations, Volume 204. Elsevier Science B.V., Amsterdam.

[4] Das, S. (2008) Functional Fractional Calculus for System Identification and Controls. Springer, Berlin.

[5] Zhou, Y. (2014) Basic Theory of Fractional Differential Equations. World Scientific, Singapore. http://dx.doi.org/10.1142/9069

[6] Wu, J. (1996) Theory of Partial Functional Differential Equations Applications. Springer, New York. http://dx.doi.org/10.1007/978-1-4612-4050-1

[7] Thandapani, E. and Savithri, R. (2003) On Oscillation of a Neutral Partial Functional Differential Equation. Bulletin of the Institute of Mathematics - Academia Sinica, 31, 273-292.

[8] Xu, R. and Meng, F. (2013) Oscillation Criteria for Neutral Partial Functional Differential Equations. Differential Equations \& Applications, 5, 69-82. http://dx.doi.org/10.7153/dea-05-05

[9] Lakshimikantham, V. and Vasundhara Devi, J. (2008) Theory of Fractional Differential Equations in a Banach Space. European Journal of Pure and Applied Mathematics, 1, 38-45.

[10] Chen, D.X. (2012) Oscillation criteria of Fractional Differential Equations. Advances in Difference Equations, 33. http://dx.doi.org/10.1186/1687-1847-2012-33

[11] Grace, S.R., Agarwal, R.P., Wong, P.J.Y. and Zaffer, A. (2012) On the Oscillation of Fractional Differential Equations. Fractional Calculus and Applied Analysis, 15, 222-231. http://dx.doi.org/10.2478/s13540-012-0016-1

[12] Chen, D.X. (2013) Oscillatory Behavior of a Class of Fractional Differential Equations with Damping. U.P.B. Sci. Bull., Series A, 75, 107-118.

[13] Feng, Q.H. and Meng, F.W. (2013) Oscillation of Solutions to Nonlinear Forced Fractional Differential Equation. Electronic Journal of Differential Equations, 2013, 1-10.

[14] Zheng, B. (2013) Oscillation for a Class of Nonlinear Fractional Differential Equations with Damping Term. J. Adv. Math. Stud, 6, 107-115.

[15] Han, Z.L. Zhao, Y.G., Sun, Y. and Zhang, C. (2013) Oscillation for a Class of Fractional Differential Equations. Discrete Dynamics in Nature and Society, 2013, 6 p.

[16] Prakash, P., Harikrishnan, S., Nieto, J.J. and Kim, J.H. (2014) Oscillation of a Time Fractional Partial Differential Equation. Electronic Journal of Qualitative Theory of Differential Equations, 15, 1-10. http://dx.doi.org/10.14232/ejqtde.2014.1.15

[17] Li, W.N. (2015) Forced Oscillation Criteria for a Class of Fractional Partial Differential Equations with Damping Term. Mathematical Problems in Engineering, 2015, Article ID: 410904, 6 p. 